\title{
Paradiplomacia: una herramienta de poder blando de China*
}

\section{Paradiplomacy: A tool of China's Soft Power}

\author{
Juan Camilo Mesa Bedoya* \\ Carlos Hernán González Parias**
}

Recibido: 15 de enero de 2016

Aprobado: 6 de julio 2016

Disponible en línea: 20 de diciembre de 2016

\section{Resumen}

El sistema internacional actual presenta un escenario interdependiente ideal para la actividad internacional de las ciudades y regiones, fenómeno denominado paradiplomacia, que cada día ocupa un lugar más significativo en el contexto académico. En este escenario, las ciudades chinas han interactuado con sus similares, en un proceso que ha tomado mayor relevancia con las reformas impulsadas por la República Popular China desde 1978. De ahí que el objetivo del artículo es demostrar que las diferentes actividades de gestión internacional realizadas por las unidades subnacionales chinas han generado que la paradiplomacia sirva como elemento complementario a las estrategias de poder blando de la política exterior del gigante asiático. El aporte del artículo es evidenciar que las ciudades chinas son actores de la política exterior del Estado y serán cada vez más representativas, no solo por

\section{Abstract}

The current international system presents an ideal interdependent scenario for the international activity of cities and regions, a phenomenon called paradiplomacy, which increasingly occupies a more significant place in the academic context. In this scenario, Chinese cities have interacted with their counterparts in a process that has become more relevant with the reforms promoted by the People's Republic of China since 1978. Hence, the objective of the article is to demonstrate that different international management activities carried out by Chinese subnational units, makes paradiplomacy useful as a complement to the soft power strategies of China's foreign policy. The contribution of the article is to show that Chinese cities are actors in the state's foreign policy and will be increasingly representative, both for their internal dynamics of urban consolidation, economic growth and

doi:10.11144/Javeriana.papo21-2.phpb

* Artículo de investigación. El artículo es resultado del proyecto de investigación Relaciones internacionales y desarrollo endógeno de la Facultad de Estudios Internacionales de la Institución Universitaria Esumer (Medellín, Colombia). ** Universidad Nacional de La Plata (La Plata, Argentina). Correo electrónico: mesabedoya@gmail.com

*** Universidad Pontificia Bolivariana (Medellín, Colombia). Correo electrónico: carlosheg@gmail.com 
sus dinámicas internas de consolidación urbana, crecimiento económico y desarrollo social, sino por el papel que se les ha dado desde el Gobierno central en el proceso de reemergencia de China como potencia global.

\section{Palabras clave}

paradiplomacia; poder blando; relaciones internacionales; política exterior; República Popular China

\section{Cómo citar este artículo:}

Mesa Bedoya, J. C. y González-Parias, C. H. (2016). Paradiplomacia: una herramienta de poder blando de China. Papel Político, 21(2), 537-563. https://doi.org/10.11144/Javeriana. papo21-2.phpb social development, and for the role the central government has given to them in China's process of re-emergence as a global power.

\section{Keywords}

paradiplomacy; soft power; international relations; foreign policy; PRC 


\title{
Introducción
}

El sistema internacional es, ante todo, dinámico y cambiante, por lo que las nuevas tendencias, los fenómenos o los actores están en la capacidad de generar cambios en él. En este sentido, Mesa, Duarte y González (2014, p. 103) indican:

La globalización, la pérdida de influencia del Estado-Nación y el surgimiento de nuevos temas en la agenda internacional, han dinamizado las Relaciones Internacionales, dando lugar a la participación de nuevos actores y a múltiples interacciones entre estos con los actores tradicionales en el sistema internacional.

Este fenómeno, que Duchacek (1986) y después Michelmann y Soldatos (1990) han definido como paradiplomacia, ha

\begin{abstract}
generado profundos cambios en el sistema internacional, propiciando nuevos escenarios en los que el Estado sigue siendo el centro, pero en el que se reconoce que es cada vez mayor la participación de actores subnacionales en el contexto internacional. (Mesa, Duarte y González, 2014, p. 103)
\end{abstract}

Del mismo modo, desde la instauración de la política de reforma y apertura en 1978 por Deng Xiaoping, China ha experimentado un proceso de descentralización administrativa en múltiples niveles, que, a la par de lo que Nye (1997) denominó la reemergencia de este país asiático, ha llevado a sus ciudades a tener cada vez más una mayor actividad en el contexto internacional.

Durante este largo periodo de reformas, China ha reconfigurado su política exterior, se ha posicionado como un importante actor en materia económica, política, cultural y militar y, al igual que las grandes potencias mundiales, ha ganado un lugar en el sistema internacional mediante combinaciones de poder duro y poder blando, usando las estrategias más convenientes según sus intereses nacionales y actuaciones coyunturales.

A partir de 2004, "desde el inicio de la 'era Hu Jintao”' (Rodríguez Arana y Leiva, 2013), se percibe con mayor fuerza la vinculación del poder blando (soft power) en la política exterior China y, de la misma manera, una mayor vinculación de las ciudades a los asuntos internacionales. En tal orden de ideas, el objetivo de este artículo es poder demostrar que la paradiplomacia, es decir, la gestión internacional de las ciudades, de la República Popular China se ha constituido en una estrategia de poder blando de la política exterior del gigante asiático.

En primera instancia, se establece un marco conceptual para definir lo que se entiende por paradiplomacia y poder blando. Posteriormente, se contextualizan los lineamientos de la política exterior de la República Popular China y se analiza el rol de las ciudades 
en los diversos asuntos del Estado, sobre todo en el interés de presentar a China "como una potencia responsable y sin pretensiones hegemónicas, cooperativa y promotora de la paz internacional” (Rocha, 2006, citado por Rodríguez Arana y Leiva, 2013, p. 501).

\section{Marco conceptual}

No puede decirse que los conceptos de paradiplomacia y poder blando tengan un mismo origen teórico. Mientras la paradiplomacia pertenece a la corriente idealista en las relaciones internacionales, el poder blando no contradice el realismo. En palabras de Nye (2011, p. 82), "el poder blando no es una forma idealista o liberal, simplemente es una forma de poder, una forma de obtener los resultados esperados", ${ }^{1}$ elevado a la categoría de herramienta de política exterior. Sin embargo, dentro de la teoría de la interdependencia compleja, se evidencian signos de que los conceptos de paradiplomacia y poder blando tienen relación y se acercan en mayor medida al idealismo, respondiendo a coyunturas similares del sistema internacional. En esta teoría, Keohane y Nye (1988) desarrollan tres pilares fundamentales: 1 ) la existencia de múltiples canales que pueden describirse como relaciones interestatales, transgubernamentales y transnacionales; 2) la emergencia de una agenda global con múltiples dimensiones donde la seguridad militar no es el único ámbito prioritario; y 3) la superación de la (necesidad de) utilización de la fuerza militar como elemento cardinal en las relaciones entre Estados.

El primero aporta el punto de partida para el posterior desarrollo teórico-conceptual de la paradiplomacia. En este marco teórico, la consideración de Keohane y Nye (1988) de que la política no se detiene al borde del agua, argumentando la cada vez más angosta distinción entre la política exterior y la doméstica ha conllevado que "las relaciones entre los actores internacionales estén cada vez más proclives a la proposición de una nueva agenda internacional, caracterizada por temas de competencia de todos los niveles de la estructura administrativa de los Estados” (Mesa, Duarte y González, 2014, p. 104).

Rosenau (1997), por su parte, ha definido este fenómeno de transformaciones tanto del sistema internacional como del papel del Estado en las relaciones internacionales como uno de fragmentación en dos direcciones: hacia arriba, es decir, hacia instancias supranacionales, como las organizaciones internacionales; y hacia abajo, es decir, en relación con grupos subnacionales, como los Gobiernos locales, lo cual ha permitido a estos últimos desarrollar "alternativas de participación en el escenario mundial y, pese a no ser considerados como sujetos del Derecho Internacional" (Calvento, Lorenzo y Herrero, 2014, p. 4), les da la posibilidad de intervenir en "modalidades de cooperación internacional, establecer o cumplir acuerdos de cuño económico y cultural, de modo formal e informal" (Vigevani, 2004, p. 28).

${ }^{1}$ Las traducciones son nuestras. 
En torno a la participación internacional de las ciudades se han desarrollado conceptos tales como diplomacia descentralizada (Rodríguez Cuadros, 2009), protodiplomacia (Paquin, 2004), paradiplomacia identitaria (Paquin, 2004), nueva diplomacia regional (Keating, 2001), política externa federativa (Rodrigues, 2004), diplomacia federativa (Bogéa, 2002), por mencionar algunos, los cuales comparten elementos comunes al concepto de paradiplomacia.

La diplomacia descentralizada es un concepto amplio en el que cabe una multiplicidad de actores que intervienen en el sistema internacional. Rodríguez Cuadros (2009) la concibe como

el conjunto de interacciones internacionales que se realizan entre los Gobiernos nacionales y Gobiernos subnacionales, entidades de la sociedad civil de otros Estados, incluidas empresas, u organizaciones no gubernamentales; entre Gobiernos subnacionales con Gobiernos subnacionales extranjeros o con entidades de la sociedad civil u organizaciones no gubernamentales extranjeras en función de intereses públicos; y, entre organizaciones no gubernamentales de Estados diferentes siempre en función de intereses o bienes públicos. (p. 59)

Esta primera definición aporta en el sentido de identificar los actores, de diferente naturaleza, que interactúan en nivel internacional, y también en el sentido de reconocer que tales interacciones son generadas gracias a la búsqueda de intereses públicos o en ejercicio de funciones públicas. Por tanto, según lo anterior, se descartan todas aquellas interacciones internacionales de entes privados, cuyos objetivos sean exclusivos de tipo privado para la organización. No obstante, como se percibe, este concepto aún está por desarrollar, dado que describe más una simple acción internacional que un proceso sistemático llevado a cabo por actores precisos y con objetivos claros.

Por otro lado, la protodiplomacia es entendida por Paquin (2004) como la actividad internacional de las entidades subnacionales con finalidades independentistas y de soberanía. Asimismo, concibe el concepto de paradiplomacia identitaria también como la acción internacional de las entidades subnacionales, pero orientada, no a la independencia, sino al fortalecimiento de identidades y a la construcción de nación en Estados plurinacionales. Para clarificar mejor los conceptos, Zeraoui (2011) explica a manera de ejemplo que el Kurdistán iraquí es un caso de protodiplomacia, puesto que las autoridades locales hacen una reivindicación al derecho a la independencia por medio de actuaciones en la esfera internacional, mientras que Cataluña, hasta septiembre de 2015 (Piñol, 2015), representaba un caso de paradiplomacia identitaria, pues el objetivo de las autoridades locales era por medio de acciones de representación internacional recobrar y reconstruir la nación catalana, por lo que concluye que "la línea que separa las dos acciones es tan débil que se puede pasar del uno al otro de una manera imperceptible" (p. 68). 
Keating (2001) concibe la nueva diplomacia regional como la actividad internacional de las regiones - en el contexto europeo- que tiene origen en procesos políticos, económicos y culturales, para la conformación de bloques supranacionales, la regionalización de la producción y por cuestiones idiomáticas, respectivamente.

Rodrigues (2004), citado por Vigevani y Figueiredo (2012, p. 180), define la política externa federativa como "la estrategia propia de un estado (federal) o municipio, desarrollada en el ámbito de su autonomía en aras de su inserción internacional de forma individual o colectiva”, con lo cual indica cierta desvinculación de la política exterior del Estado en el que se encuentra. Sin embargo, Bogéa (2002), citado por Vigevani y Figueiredo (2012, p. 180), difiere de este concepto y formula la diplomacia federativa por medio del cual la acción internacional subnacional "desarrolla un nuevo modelo de diplomacia que está condicionada a las necesidades del poder central y complementa a los Gobiernos centrales en la identificación de nuevas oportunidades internacionales para la cooperación, el comercio y la atracción de inversiones”.

Ahora bien, una vez clarificados los conceptos que tratan la actividad internacional de los Gobiernos locales bajo diferentes perspectivas, se aborda el concepto de paradiplomacia y se justifica la causa de utilización de este en lugar de los anteriormente desarrollados.

Como se mencionó, el concepto de paradiplomacia desarrollado por Duchacek (1986), Michelmann y Soldatos (1990), citados por Schiavon y Velázquez (2008), consiste en

los contactos, actividades, procesos e iniciativas externas entre gobiernos no centrales (estados federados, provincias, cantones, länders, etcétera) y otros actores internacionales, tales como los Estado-naciones, gobiernos locales similares [...]. Estas actividades no representan la política exterior de un Estado puesto que esta es una atribución exclusiva de los gobiernos centrales. Pero se trata de acciones que complementan y apoyan la política exterior puesto que el sufijo "para" significa "paralelo", "junto a” o "asociado. (p. 27)

En ese sentido, la actividad internacional de los Gobiernos locales, dentro de la paradiplomacia, no compite con la diplomacia estatal; al contrario, se convierte en una herramienta de potenciación y fortalecimiento de los asuntos domésticos y de la política exterior del Estado, porque "básicamente las entidades buscan una vinculación externa para mejorar su desarrollo u obtener un beneficio local, entre otros objetivos" (Zeraoui, 2011, p. 77), de corte económico, político y cultural. En el ámbito económico, es notable la búsqueda del crecimiento mediante la captación de cooperación internacional descentralizada en sus diferentes modalidades, la atracción de inversión extranjera, la promoción del turismo y la identificación de mercados extranjeros para la colocación de bienes y servicios de producción local. 
En el aspecto cultural, "los Gobiernos locales tratan de establecer convenios de intercambio cultural, educativo y artístico con otras regiones del mundo" (Schiavon y Velázquez, 2008, p. 32).

La variable política es desarrollada mediante la firma de convenios, acuerdos y declaraciones de hermanamientos que buscan "promover los contactos culturales y fomentar los enlaces humanos, sin contradecir o interferir de alguna forma con la política exterior del Estado al que conforman" (Zeraoui, 2011, p. 88). Zaharna (2009, p. 95) considera que los hermanamientos son acuerdos que ayudan a promover "la cooperación y los intercambios en una variedad de áreas tales como la economía, el comercio y el turismo, así como la educación, la tecnología y los deportes [...] que sirven para institucionalizar el proceso de construcción de relaciones”. En general, las ciudades realizan hermanamientos sobre elementos que comparten: "proximidad geográfica; vínculos históricos, culturales o comerciales; una comunidad migrante del país de origen; incluso un problema para el que comparten mentes y experiencia en su solución” (Jain, 2005, p. 66), por lo que se espera que los beneficios sean mutuos, aunque no necesariamente en la misma cantidad para las partes.

Los hermanamientos han sido ampliamente utilizados por las ciudades con diferentes propósitos. Desde uno de los primeros hermanamientos que data de 836, entre las ciudades de Paderborn (Alemania) y Le Mans (Francia), hasta, por ejemplo, ciudades de Estados con poca antigüedad que también han generado hermanamientos, como Dubái (Emiratos Árabes Unidos), que cuentan con quince hermanamientos, entre los que se incluyen ciudades como Osaka, Estambul, Ginebra, Shanghái y Detroit (Zaharna, 2009, p. 95).

No obstante, también se debe señalar que los hermanamientos

pueden ser una mera declaración de intenciones, que se plasma en la firma de un acuerdo de amistad, sin pasar de ahí. Puede plasmarse en una partida presupuestaria de una entidad hacia la otra [...] o en su máxima expresión puede buscar un acercamiento más amplio entre las sociedades. (Rodríguez García, 2013, p. 276)

Lo anterior depende, en gran medida, de las administraciones locales que son las encargadas de dinamizar y materializar dichos hermanamientos.

En ese orden de ideas, la paradiplomacia es el concepto que encaja de manera perfecta con el objeto de estudio del presente artículo, toda vez que se enfoca en la actividad internacional de las ciudades, pero no en una actividad aislada, sino en una gestión internacional enfocada, direccionada y amparada por la política exterior del país al que están adscritos, entendiendo que "la formulación de política exterior es en general competencia de los Estados, pero estos no son los únicos agentes" (Llenderrozas y Finkielsztoyn, 2013). 
Retomando los pilares de la interdependencia compleja, el segundo y tercer pilar se refieren a un vaciamiento del poder por la vía militar - que no significa una negación de su importancia ni tampoco una negación de que continúa siendo usada por los Estados - hacia nuevos espectros del poder, en los que la capacidad de influir en el otro está supeditada a aspectos económicos, políticos y culturales.

Continuando con esta misma línea conceptual, la característica de las interdependencias de tener un carácter asimétrico y las manifestaciones de estas asimetrías en los aspectos mencionados (economía, política y cultura) son una importante fuente de poder que reestructura su naturaleza, su distribución y ejercicio (Keohane y Nye, 1988), lo cual genera una nueva disposición. Ahora el poder "no se sostiene únicamente en la supremacía militar de las potencias hegemónicas, sino en ámbitos estructurales que definen las normas de relaciones entre los actores del sistema internacional antes que estas mismas relaciones se produzcan” (Millán, 2013, p. 681).

En ese orden de ideas, Del Arenal (2009), citado por Millán (2013) expresa:

El poder ya no es sólo un fenómeno relacional sino también y cada vez más, como consecuencia de la creciente interdependencia, un fenómeno estructural, mucho más sutil, consistente en el control o la capacidad para influir o determinar las estructuras y dinámicas del sistema o conseguir que los demás quieran lo que uno quiere. (p. 211)

Siguiendo esta connotación de las capacidades de los Estados para influenciar en otros por vías diferentes de las militares, Strange (1998) desarrolla el concepto de poder estructural considerándolo como "la potestad de decidir cómo serán hechas las cosas, la potestad de configurar marcos dentro de los cuales los Estados se relacionarán entre sí, se relacionarán con los individuos o se relacionarán con las empresas” (p. 25). En ese sentido, desarrolla la concepción de que el poder es algo más que "la capacidad para establecer la agenda internacional o para diseñar regímenes internacionales" (Sodupe, 2002, p. 93). Así, Sanahuja (2007), citado por Millán (2013, p. 682), señala:

El poder estructural radica en la capacidad de definir de antemano los costes de oportunidad de cada opción y la consiguiente estructura de incentivos y sanciones, así como de asignar funciones y determinar qué conductas son aceptables, y cuáles no, en función de unos valores determinados. (p. 334)

El poder duro (hard power) y el poder blando (soft power) son dos caras de la misma moneda: la manifestación de poder de los Estados. El primero se considera un poder directo por las capacidades de "inducir a terceros a cambiar de posturas" (Nye, 2003) por la vía militar y económica, mientras que el segundo es un poder indirecto, ya que, 
sin la utilización de la fuerza, se tiene la capacidad "para moldear los valores, intereses y motivaciones de otros actores" (Millán, 2013, p. 682). El poder blando es "la aptitud para obtener lo que se quiere o se desea mediante el poder de atracción y la seducción en lugar de la fuerza o el dinero" (Nye, 2003, citado por Pereira, 2008). Resalta este autor que el poder blando "brota del atractivo de la cultura, de los ideales y valores políticos y de la legitimidad y autoridad moral de la política exterior”.

Para relacionar ambos conceptos, paradiplomacia y poder blando, vale la pena resaltar que, como la paradiplomacia no se ocupa de los asuntos de primer nivel, tales como la seguridad nacional, la soberanía y la firma y ratificación de tratados internacionales, los cuales son monopolio absoluto de los Estados, su connotación está más ligada con asuntos de segundo nivel, como la cooperación y la atracción de inversión. En ese sentido, y en la medida en que sea fomentada, se convierte en una herramienta de los Estados para generar alternativas de poder blando y coadyuvar al logro de sus objetivos estratégicos, en connotación de la hipótesis desarrollada por Nye bajo la idea de que el poder blando es cada vez más importante, dado que el proceso de globalización -que conlleva, entre otros, el florecimiento de nuevos actores internacionales- y el desarrollo de nuevas tecnologías de comunicación aumentan la necesidad de credibilidad en el escenario internacional y hace que el mundo sea cada vez más interdependiente.

Como ejemplo de lo anterior, sobresale el papel del presidente estadounidense Dwight D. Eisenhower en la creación de la iniciativa ciudades hermanas (sister cities) en 1956, para fomentar la paz y la prosperidad mediante el establecimiento de lazos entre personas de ciudades de todo el mundo. Se destacan de esta iniciativa la generación de un primer hermanamiento entre las ciudades de Seattle y Tokio, con el fin de reparar las tensiones posteriores a la Segunda Guerra Mundial mediante la creación de proyectos de intercambios culturales y educativos y, posteriormente, la generación de amistades duraderas, así como el papel que tuvo el hermanamiento de ciudades en los momentos en los que la diplomacia estatal no había abierto canales oficiales con otros Estados, como en el caso de China, país con el que se establecieron hermanamientos a finales de la década de 1970 (SiterCities International, 2006). Por lo cual, puede interpretarse una función de la paradiplomacia dentro de las estrategias de poder blando de la política exterior norteamericana.

\section{Lineamientos de la política exterior de la República Popular China}

Russet y Starr (1989), citados por Pérez Gil (2012, p. 94), hacen hincapié en que la "política exterior es la actuación del Estado en el sistema internacional”; sin embargo, no debe entenderse como una actuación aislada: la política exterior de un Estado -y en mayor medida la de las potencias - integra toda una estrategia y una forma de actuar en el sistema internacional y desarrolla los fines y objetivos puntuales de su cosmovisión y de su manera de entender el mundo. 
Calduch (1993, p. 4) entiende la política exterior como una parte de "la política general formada por el conjunto de decisiones y actuaciones mediante las cuales se definen los objetivos y se utilizan los medios de un Estado para generar, modificar o suspender sus relaciones con otros actores de la sociedad internacional", resaltando que, si bien "la política exterior, la interior y la de seguridad y defensa son partes de la política general del Estado, la diferencia fundamental radica en los destinatarios: la política exterior, y solo ésta, se dirige a Estados y otros actores internacionales” (Pérez Gil, 2012, p. 98).

Cada Estado, entonces, elabora su política exterior centrado en la visión de su papel e interés de representación en el mundo. En el caso de la República Popular China, la han definido como una "política exterior de paz bajo el principio de independencia y autodecisión" (Ministerio de Relaciones Exteriores de China, 2003).

En términos generales, esta política ha tenido fluctuaciones interesantes desde la proclamación de la República Popular China en 1949 por parte las fuerzas del Partido Comunista. En esta dirección, Wang (2000) brinda una pertinente clasificación, basada en la doctrina de política exterior dominante en China en periodos particulares.

La primera etapa comprendida desde los primeros años posteriores a la revolución hasta inicios de la década de 1960 se denominó doctrina de liangge zhenying, la alianza con la antigua Unión Soviética en contra de los Estados Unidos. Sin embargo, con la llegada de Krushev al poder, se rompió la alianza chinosoviética por temas delicados, como el nulo apoyo soviético a los bombardeos en Taiwán y en la relación con el movimiento separatista del Tíbet (León de la Rosa, 2015), a causa de esto China se embarcó en un periodo de aislamiento internacional, en el que el radicalismo maoísta se afianzó y se impulsó la Revolución Cultural durante la década de 1960. La doctrina que guía esta segunda etapa denominada fandui di xiu fan comprendió buena parte de aquella década y elevó como principio rector de la política exterior china la oposición tanto al imperialismo norteamericano como al revisionismo soviético.

Iniciando la década de 1970, el contexto nacional e internacional y el acercamiento de China con los Estados Unidos trajeron consigo una nueva doctrina en la política exterior del país asiático llamada sange shijie, que es considerada como el reingreso de China al escenario internacional. Según León de la Rosa (2015), fue importante, ya que

\footnotetext{
lograría dos cosas que serían la base para el fenómeno chino actual: el retiro del apoyo estadounidense a Taiwán y por lo tanto el reconocimiento de la República Popular China como único Gobierno chino; por otro lado, la posesión de una silla en el Consejo de Seguridad de la Organización de las Naciones Unidas. (p. 24)
}

En esta etapa, China también ingresa a diversas organizaciones internacionales e inicia, tras la muerte de Mao Zedong, el periodo de reformas de Deng Xiaoping, quien 
institucionaliza y estructura la política exterior del país y la fundamenta valiéndose de la frase: "Lo chino para la esencia, lo occidental para lo práctico". La cuarta etapa, desde la década de 1980, cuando se estableció la doctrina heping yu fazhan, donde la política exterior china era orientaba a evitar alianzas estratégicas y la búsqueda de una mayor participación en la economía mundial (Wang, 2000, pp. 478-479).

Los lineamientos planteados en aquel entonces continúan presentes en la política exterior de China hasta el inicio del periodo de $\mathrm{Hu}$ Jintao en 2004, quien diversifica las relaciones internacionales del país con el resto del mundo en "dos sentidos: en lo político, a través de la tesis de los tres mundos y, en lo económico, por la emergencia de China como demandante de materias primas y como exportador de manufacturas" (León de la Rosa, 2015, p. 25). Lo anterior puede resumirse en la figura 1.

\section{Figura 1. Evolución de la política exterior China.}

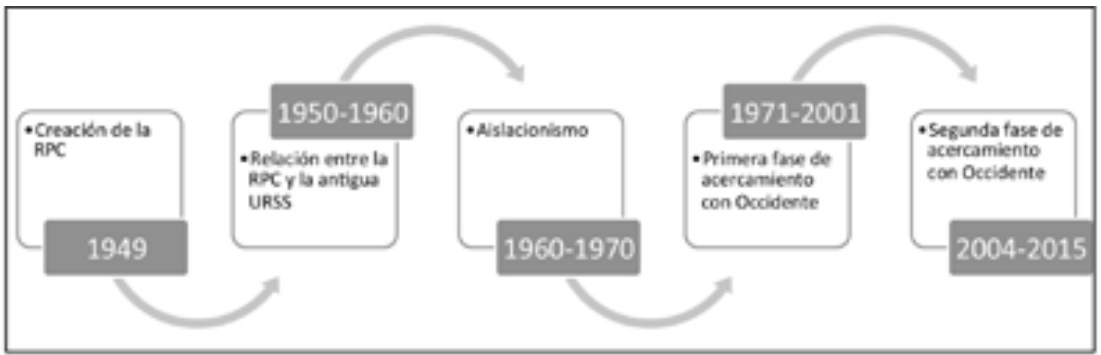

Fuente: Elaboración propia a partir de León de la Rosa (2015, p. 21).

Ahora bien, un común denominador en las diferentes etapas y doctrinas mencionadas de la política exterior China es el continuo establecimiento de relaciones bilaterales (figura 2).

Figura 2. Establecimiento de las relaciones bilaterales chinas.

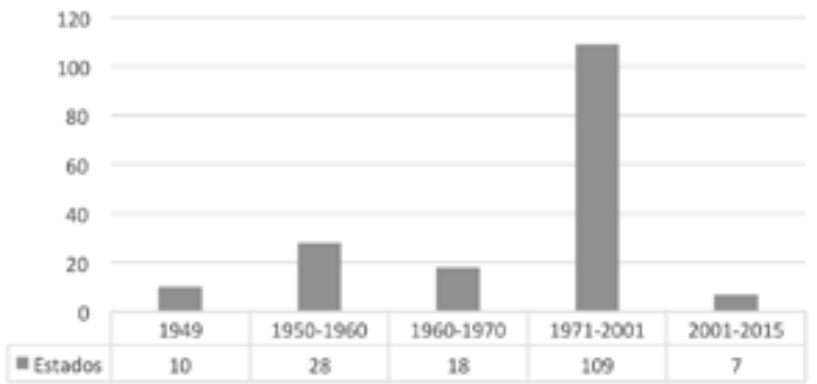

Fuente: Elaboración propia a partir de León de la Rosa (2015, p. 21) y Ministerio de Relaciones Exteriores de China (2015a). 
La política exterior china se fundamenta en cuatro pilares: 1) el mantenimiento de la integridad territorial, 2) el reconocimiento como único Gobierno chino por parte de la comunidad internacional, 3) propiciar el desarrollo económico del país y 4) incrementar su prestigio en el ámbito internacional. Para el desarrollo de los lineamientos de su política exterior y tras años de concentrarse en el poder duro, China comienza una incorporación paulatina de estrategias de poder blando para el cumplimiento de sus objetivos, introduciéndolo, en palabras de Rodríguez Aranda y Leiva (2013, p. 4),

de manera explícita en el discurso y en la política exterior a través de la diplomacia pública -otro concepto nuevo en China, y que se irá desarrollando paralelamente al de soft power - como una forma de lograr un poder comprehensivo que combine hard y soft power, además de servir como instrumento para mejorar la imagen del país en el exterior.

Esta actuación de política exterior trae consigo dos conceptos centrales durante el gobierno de Hu Jintao: 1) el ascenso pacífico ${ }^{2}$ y 2) el mundo armonioso. El primero, introduciendo la noción de China como una "potencia emergente pero responsable; China debía presentarse como un gran poder pacífico, no amenazante, pero sin renunciar a los derechos y responsabilidades que le aguardan por su nueva posición en el mundo" (Rocha, 2006, p. 706). El segundo "tiene su origen en la filosofía de Confucio, quien expuso que a pesar de que el mundo está lleno de diferencias y contradicciones, el hombre honrado debe equilibrarlas y conseguir la armonía” (Rodríguez Aranda y Leiva, 2013, p. 5), y desarrolla cinco principios: 1) una estrategia ganar-ganar, 2) el desarrollo pacífico, 3) el respeto a la diversidad, 4) la cooperación y coordinación y 5) la coexistencia pacífica, con lo cual crea un nuevo concepto de seguridad basado en la confianza, el beneficio, la igualdad y la colaboración mutua, que respeta el derecho de cada país de escoger su propio sistema social y económico de acuerdo con su realidad nacional (Shixue, 2008, p. 33). Ello con la finalidad de "mejorar la imagen de China en el exterior, eliminando prejuicios y temores que le impidan al gigante asiático consolidarse como una potencia regional y global responsable y confiable (Rodríguez Aranda y Leiva, 2013, p. 5).

Para Shixue (2008, p. 27), las prioridades de la política exterior china han evolucionado considerablemente durante la segunda mitad del siglo XX y los inicios del siglo XXI, claramente por el rápido crecimiento económico que le está brindando a China una transformación en su papel en el mundo. En ese sentido, complementa Rubiolo

\footnotetext{
${ }^{2}$ Desarrollo pacífico se dio en los principios de la Pancha Shila, cinco principios de la coexistencia pacífica, formulada en 1954 en el tratado entre la India y China que se convirtieron en la base ideológica para el movimiento de los países no alineados y para la doctrina oficial de la política exterior china. Los principios sobre los cuales se construyó eran: 1. El respeto mutuo a la integridad territorial y la soberanía. 2. La no agresión mutua; 3. La no intervención en los asuntos internos. 4. La coexistencia pacífica. 5. El beneficio mutuo e igualdad (González y Mesa, 2016).
} 
(2010, p. 163): "El proceso de modernización económica de China fue acompañado de una nueva orientación en la política exterior centrada en la apertura y en la búsqueda de una mejor inserción en el sistema internacional".

Desde el inicio de la era de $\mathrm{Hu}$ Jintao en 2004, el discurso y las acciones de poder blando se introducen y desarrollan en la política exterior china. Precisamente, una de las estrategias de poder blando es la difusión cultural. Autores como Zhongying (1997), Jiru (1999) Cho y Jeong (2008) y Glaser y Murphy (2009) concuerdan en que la cultura china es el principal recurso para esta forma de poder.

Uno de los símbolos de esa estrategia lo constituye la difusión de los institutos Confucio por el mundo. El primero de ellos instaurado en Seúl, el 21 de noviembre de 2004, y desde ese momento a 2016, existen cerca de 1106 alrededor del mundo. La figura 3 muestra el número de institutos Confucio por continente y los países por continente en los que hace presencia y la figura 4 la ubicación geográfica.

Figura 3. Institutos Confucio.

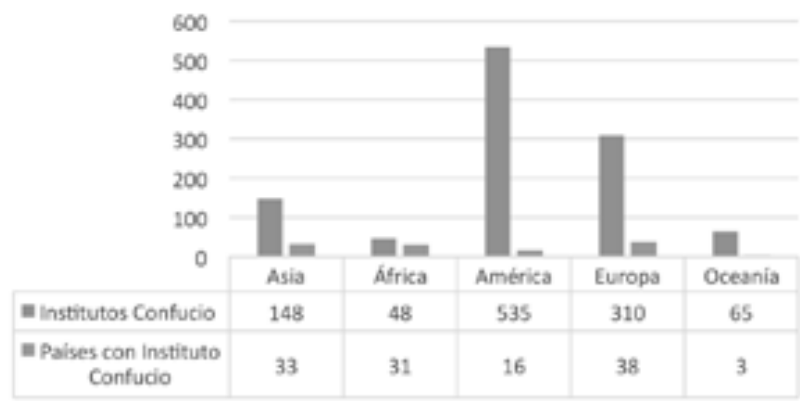

Fuente: Elaboración propia a partir de Confucius Institute (2016).

Figura 4. Institutos Confucio en el mundo.

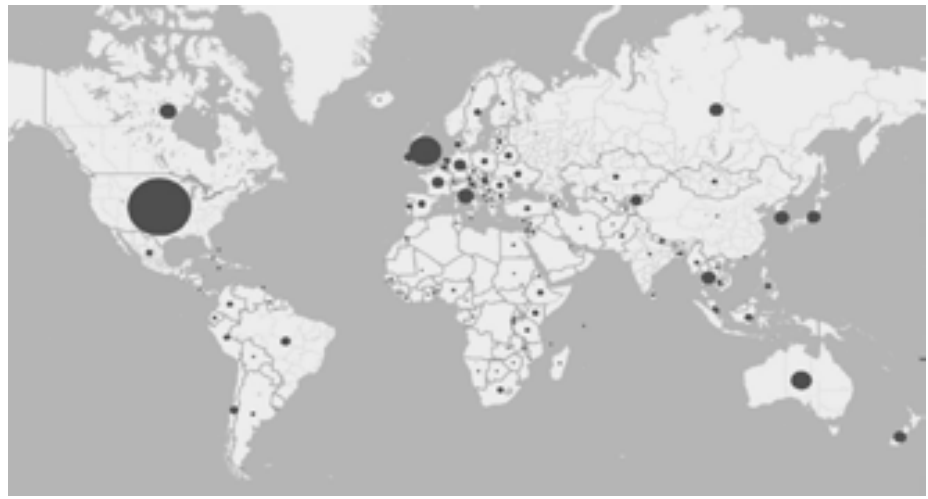

Fuente: Elaboración propia a partir de Confucius Institute (2016). 
Es de resaltar que, mediante los institutos Confucio, China hace presencia cultural en 121 países, de los 172 con los cuales tiene relaciones bilaterales, y que, a pesar de ser una estrategia clara de su política exterior, los institutos se vinculan a universidades o centros educativos y no al sector educativo y cultural gubernamental.

Ahora bien, una vez establecidos los lineamientos de la política exterior de la República Popular China, se analiza la actividad internacional de sus ciudades en relación con los objetivos puntuales de consolidación regional y mundial del gigante asiático.

\section{Paradiplomacia de las ciudades chinas}

La actividad internacional de las ciudades chinas, es decir, la paradiplomacia, es un fenómeno que las ha llevado a tener cierta relevancia en la escena internacional. En primer lugar, por el desarrollo alcanzado por algunas de ellas (Pekín y Shanghái ocuparon los puestos 9 y 21 en el Global Cities Index [ATKearney, 2015]) y su posición geográfica; y en segundo lugar, por las estrategias de consolidación del país desde las acciones de política exterior.

Con la talla de un país europeo intermedio, las provincias y las grandes ciudades chinas, al calor de las reformas, han multiplicado sus relaciones internacionales, al principio en el plano económico y cultural, y después de forma más global, y han puesto en marcha su propia diplomacia (Cabestan, 2010).

Por tanto, es asumible que la gestión internacional de las ciudades chinas está ligada, como se explicó en el marco conceptual, a los planos en los que tienen competencia, es decir, los asuntos de segundo nivel; sin embargo, en muchos casos, y por direccionamiento del poder central, han tenido relevancia en asuntos de primer nivel, como cooperación con otros Estados o presencia de delegaciones de las ciudades y regiones en organismos intergubernamentales y supranacionales.

Como ejemplos de ello, podemos citar la localización en Nanning, la capital de Guanxi, de acciones dirigidas a fomentar las relaciones de China con la Asociación de Naciones del Sudeste Asiático; de las ciudades de Yunnan (en especial Kunming) en relación con Birmania; de Changchun y otras ciudades de Manchuria en cuanto a la cooperación con Rusia o con Corea del Norte; de Ürümqi en lo referente a las zonas fronterizas de Asia Central; o de las ciudades de Shandong en relación con Corea del Sur (Niño, 2014).

Estas acciones, como se ha mencionado, no son aisladas, sino que corresponden a estrategias lideradas por el Gobierno chino y encaminadas a objetivos específicos.

Lo anterior es posible afirmarlo, dado que en la estructura gubernamental de China existe todo un andamiaje administrativo para la paradiplomacia: las grandes ciudades cuentan con departamentos de relaciones internacionales, los cuales son direccionados desde el Ministerio de Asuntos Exteriores; estos departamentos desarrollan funciones consulares, como emisión de pasaportes y visado, y atienden las delegaciones consulares de otros países; realizan hermanamientos y acuerdos de cooperación con 
ciudades extranjeras y pertenecen a asociaciones de amistad alrededor del mundo. Para eventos especiales, como los Juegos Olímpicos de 2008 y la Exposición Universal de 2010, los departamentos de relaciones internacionales de Pekín y Shanghái fueron encargados de la atención de delegaciones especiales provenientes del exterior.

Para el análisis, y sin desmeritar las demás funciones de los departamentos de relaciones internacionales de las principales ciudades chinas, se da prioridad a la función de realización de hermanamientos, acuerdos de cooperación y participación en asociaciones de amistad, toda vez que estas tienen mayor relación con los objetivos en materia política, económica y cultural que se desarrollan con la paradiplomacia y el poder blando de la República Popular China.

Las ciudades chinas mantienen una gran cantidad de contactos con ciudades extranjeras. Estas se concretan en visitas y misiones oficiales, visitas de estudio, encuentros entre Alcaldes, responsables políticos y delegaciones empresariales auspiciadas por ciudades. (Niño, 2014, p. 12)

Estas acciones luego se institucionalizan y oficializan ante los Gobiernos centrales por medio de hermanamientos o acuerdos de cooperación.

Para noviembre de 2015, la China International Friendship Cities Association (CIFCA) registra $2258^{3}$ hermanamientos de ciudades chinas con ciudades del exterior del país. Este proceso no ha sido directo, sino que ha registrado un crecimiento paulatino: mientras que para 1978, al inicio de la era de Deng Xiaoping, se registraban cinco hermanamientos de ciudades, con la política de apertura y reforma se aumenta este número hasta 1144 hermanamientos, al inicio de la era de $\mathrm{Hu}$ Jintao en 2004, periodo en el que posteriormente se registran otros 1114 hermanamientos de ciudades, tal como se observa en la figura 5 .

Figura 5. Hermanamiento de ciudades chinas desde 1973.

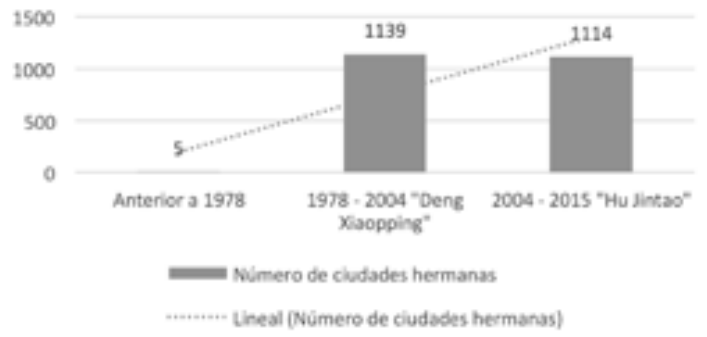

Fuente: Elaboración propia a partir de datos de CIFCA (2015).

${ }^{3}$ Esta cifra fue consultada por última vez el 4 de enero de 2016. Sus variaciones corresponderán a futuras actualizaciones por firma de nuevos hermanamientos. 
Puede asumirse que esta actividad de paradiplomacia de las ciudades chinas es una clara muestra del interés desde el Gobierno central por el posicionamiento de sus ciudades en la esfera global, dado que el incremento de los hermanamientos no solo se da por el claro fenómeno de la globalización, sino que se han incrementado exponencialmente con los periodos de grandes reformas y aperturas y con el mayor peso de las estrategias de poder blando dentro de la política exterior, puesto que, desde la era de $\mathrm{Hu}$ Jintao, en apenas nueve años, se han generado casi los mismos hermanamientos que durante veintiséis años.

Dicho proceso obedece a dos factores principalmente: uno de tipo interno y el otro externo. Del tipo interno a causa de la ampliación del campo de acción geográfica de la política exterior de China a nuevas latitudes como América Latina y África. El factor externo obedece al aumento de la importancia creciente del ejercicio paradiplomático en el mundo, donde cada vez más ciudades buscan hermanamientos con sus similares de otros Estados.

Por tanto, si este proceso continúa de la misma manera, es presumible que el número de hermanamientos de las ciudades chinas supere los 3000 en menos de cinco años.

\section{Figura 6. Hermanamientos de ciudades chinas en el mundo.}

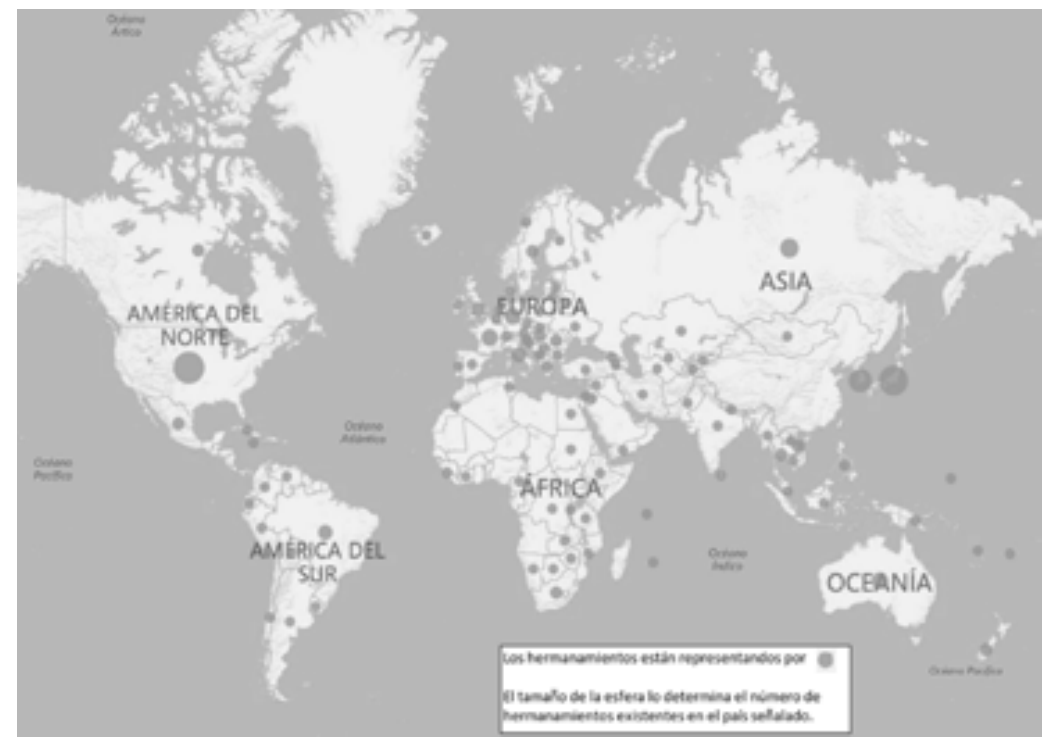

Fuente: Elaboración propia a partir de datos de CIFCA (2015).

Como se observa en la figura 6, los hermanamientos de las ciudades chinas se extienden alrededor del mundo. Si bien cada ciudad y su respectiva hermana tienen características y objetivos particulares, en la mayoría de las ocasiones los hermanamientos se dan con ciudades con características similares, en aspectos sociales, culturales, políticos y económicos. Tal es el caso de las ciudades de Pekín y Shanghái, reconocidas por ser la 
capital política y económica de China, respectivamente, en las que los hermanamientos tienen lugar con ciudades afines a sus intereses particulares; en ese sentido, Pekín tiene hermanamientos con ciudades capitales como Washington, D. C., Ottawa, Canberra, Berlín, París, Madrid, Londres, Wellington, Tokio, Ankara e Islamabad, claramente en fortalecimiento de un interés político, mientras que Shanghái mantiene hermanamientos con ciudades con gran importancia económica, que muchas veces coincide también con intereses políticos, tales como Budapest, Maputo, Manila y Londres.

Una característica adicional que tienen los hermanamientos de las ciudades chinas es la materialización de dos de los lineamientos de la política exterior del país: el mantenimiento de la integridad territorial y el reconocimiento como único Gobierno chino; para tal fin, la orientación geográfica de los hermanamientos ha tenido como punto de partida el continente asiático en la región nororiental, en clara actuación paralela de la atención prioritaria de la política exterior de China hacia esta región, y posteriormente se ha ido escalonando hacia ciudades europeas, americanas, de Oceanía y, en menor medida, africanas. Es importante destacar, en este apartado, que se analizaron algunos de los documentos que soportan los convenios de hermanamiento y todos tienen una estructura similar, una suerte de copia o formato, replicable a todas las ciudades, lo cual sugiere una estrategia estructurada y direccionada, que sustenta la tesis de que la paradiplomacia, cuando es incentivada por el Gobierno central, es una herramienta para las estrategias de poder blando dentro de la política exterior.

Para el desarrollo de los dos últimos lineamientos de la política exterior de China, propiciar el desarrollo económico del país e incrementar su prestigio en el ámbito internacional, y siguiendo esta misma línea, durante 2012 y 2013 se celebraron en Pekín las 15 y 16 cumbres China-Unión Europea, destacándose entre ellas el primer encuentro entre alcaldes de ciudades chinas y de la Unión Europea y la firma de acuerdos de cooperación sobre urbanización y desarrollo sostenible.

En relación con la India, se ha establecido un marco de acuerdos provinciales y municipales que marca hitos en las relaciones diplomáticas entre los dos Estados por medio de los hermanamientos de Pekín con el estado de Delhi, Chengdu con Bangalore y Kunming con Calcuta.

Por otro lado, es destacable el acuerdo firmado por la ciudad China de Chengdu con Łódź en Polonia, para la cooperación en aspectos de transporte en la posible construcción de un ferrocarril para comunicar China con Europa Central y Oriental. En ese orden de ideas,

se ha podido comprobar cómo la cooperación entre ciudades ha formado parte de las negociaciones de China con la Unión Europea, con la India y con el bloque de países de Europea Central y Oriental. En cada uno de esos tres casos, se han producido iniciativas 
relativas al ámbito urbano y de las ciudades que han contribuido a fortalecer el diálogo político. (Niño, 2014, p. 15)

Al observarse el plano global, la paradiplomacia y la política exterior china sugieren marcar un mismo paso, o en términos más técnicos, direccionarse bajo unos mismos lineamientos que tengan alcances similares desde el punto de vista de la difusión. Tras observar la figura 6 y analizar los papeles que han desempeñado las ciudades en escenarios particulares, se destaca que, mientras China sostiene relaciones diplomáticas con 172 países, y ha establecido 162 misiones diplomáticas oficiales en diferentes lugares del mundo, las ciudades de este Estado mantienen hermanamientos con sus homólogas de 132 países. Esto es una clara muestra de la expansión que hace China de las combinaciones de poder duro, como en el caso de las islas Diaoyu (en chino) o Senkaku (en japonés) en 2012, la construcción de islas artificiales en el mar del Sur de China durante 2015 o las posturas no negociables internacionalmente frente a Taiwán, el Tíbet y Xinjiang (Sinkiang) y poder blando en el sistema internacional, pues combina métodos tradicionales en el ejercicio de las relaciones internacionales y la diplomacia, con acciones no tradicionales de política exterior; lo importante es que se mantiene en su objetivo de incrementar la presencia en el contexto internacional.

\section{Figura 7. Comparativo: relaciones bilaterales chinas, países con misiones diplomáticas chinas y países con hermanamientos de ciudades chinas.}

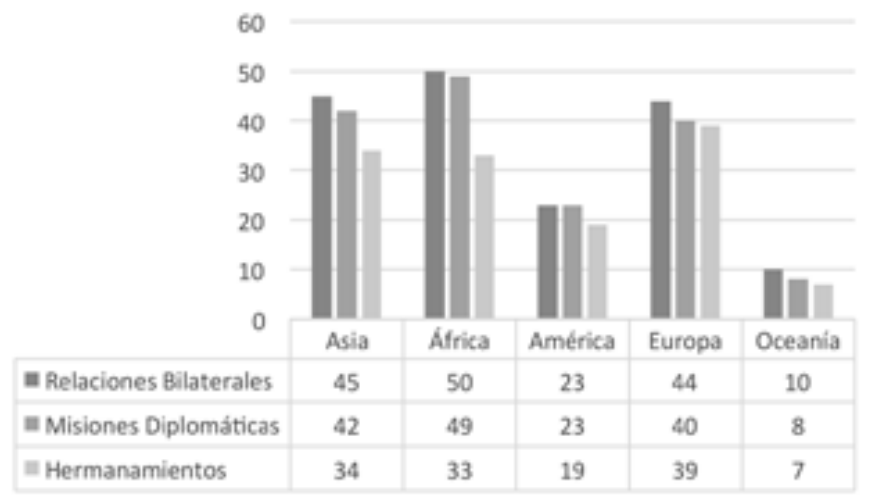

Fuente: Elaboración propia a partir de CIFCA (2015) y Ministerio de Relaciones Exteriores de China (2015b).

La figura 7 permite observar la distribución geográfica de las relaciones bilaterales, de las misiones diplomáticas y de los hermanamientos realizados por los entes subnacionales chinos. En primer lugar, se evidencia que las zonas geográficas donde la 
República Popular China posee mayor presencia diplomática corresponden también al mayor número de hermanamientos.

Por otra parte, se encuentra correlación con respecto a las zonas de influencia y estratégicas para el gigante asiático: Asia donde China es llamada a consolidarse como líder natural de buena parte de este diverso continente y África reviste para China una enorme importancia geoestratégica explicada especialmente a causa del aumento tanto de alimentos como de materias primas, las cuales son características de buena parte de las economías africanas.

La rápida penetración de China en los mercados africanos tiene mucho que ver con su promoción deliberada de una política exterior libre de servidumbres políticas, que, sumada a la buena disposición de Pekín a proporcionar ayuda y préstamo a bajo interés, se ha mostrado enormemente atractiva para los dirigentes africanos. China, que dispone de capital abundante y experiencia tecnológica y se siente en posesión de una particular legitimación política, se ha embarcado en una búsqueda planetaria de recursos sin explotar, nuevos mercados y socios diplomáticos fiables, en la que África figura en lugar destacado (Alden, 2007, p. 17).

Ahora bien, si se observa porcentualmente con respecto a los totales respectivos de cada variable analizada, la pequeña diferencia que existe no se percibe con claridad (figura 8).

\section{Figura 8. Comparativo porcentual: relaciones bilaterales chinas, países con misiones diplomáticas chinas y países con hermanamientos de ciudades chinas.}

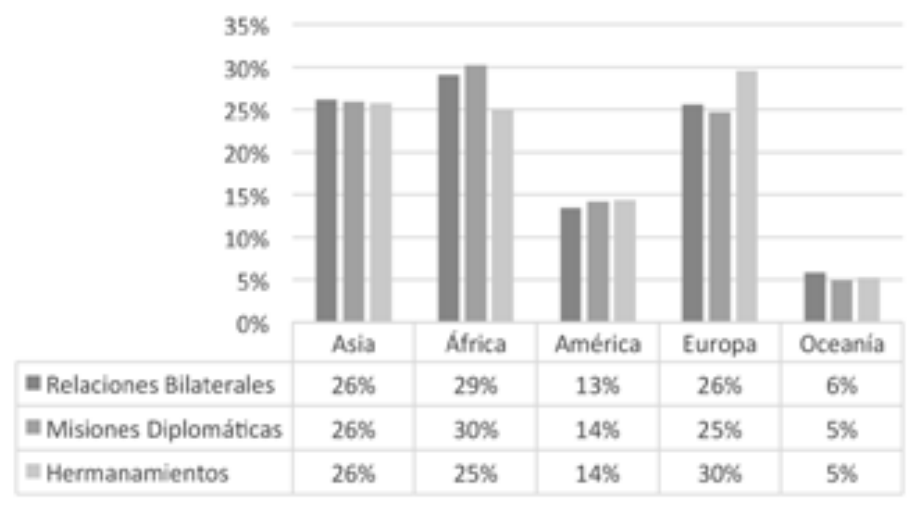

Fuente: Elaboración propia a partir de CIFCA (2015) y Ministerio de Relaciones Exteriores de China (2015b).

Como se puede observar, la relación de hermanamientos, misiones diplomáticas y relaciones bilaterales de China en el continente asiático, americano y oceánico es similar para cada variable; sin embargo, se observa un fenómeno particular para África y Europa: 
mientras en el primero existe menos cantidad de hermanamientos respecto de las otras dos variables, en el segundo los hermanamientos superan en número la acción de la política exterior oficial de China en el continente. Respecto de Europa, se explica principalmente a causa de la importancia histórica que han tenido los hermanamientos en la misma construcción de la Unión Europea, las cuales, después de la Segunda Guerra Mundial, fueron una herramienta para lograr la reconciliación social entre los Estados beligerantes y hoy por hoy son empleados hacia países por fuera del continente, con el fin de "crear relaciones de confianza y comprensión mutua” (Conseil des Communes et Régions d'Europe, 2008, p. 2).

Ahora bien, si se analiza cronológicamente la firma de hermanamientos de ciudades chinas con el establecimiento de relaciones bilaterales de los respectivos países a los que pertenecen esas ciudades, es clara la existencia de una tendencia a disminuir los periodos en los que se firmaron hermanamientos posteriormente al establecimiento de relaciones. Por ejemplo, mientras la República Popular China estableció relaciones bilaterales con Mongolia en octubre de 1949, el primer hermanamiento de una ciudad china (Hohhot) con una ciudad mongola (Ulan Bator) no sucedió hasta octubre de 1991; o en el caso de la India, en el que se establecieron relaciones bilaterales en enero de 1950, el primer hermanamiento entre una ciudad china (Kunming) y una india (Calcuta) no sucedió hasta octubre de 2013, sesenta y tres años después. Sin embargo, en casos como el de los Estados Unidos, país con el que China estableció relaciones bilaterales en enero de 1979, la firma de hermanamientos entre ciudades (NanjingSan Luis) se comenzó a realizar al mes siguiente; o en el caso de Sudáfrica, país con el que se establecieron relaciones en enero de 1998 y se firmaron hermanamientos entre ciudades (Pekín-Johannesburgo) en junio de ese mismo año.

\section{Figura 9. China: establecimiento de relaciones bilaterales- acuerdos de hermanamiento entre ciudades.}

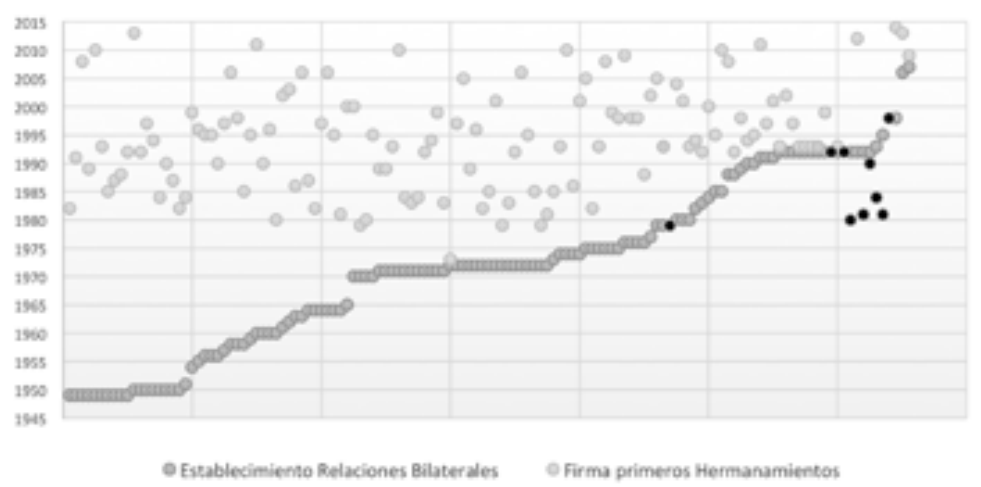

Fuente: Elaboración propia a partir de CIFCA (2015) y Ministerio de Relaciones Exteriores de China (2015b). 
Bajo esa lógica, la figura 9 muestra la relación entre el año en que China estableció relaciones bilaterales con un país (puntos en gris oscuro) y el año en que se establecieron hermanamientos de ciudades chinas con el país determinado (puntos en gris claro). Si bien China mantiene relaciones con 172 Estados, en la figura se muestran 132 en relación con los países a los que pertenecen las ciudades con las que se tienen hermanamientos. Como se puede observar a partir de la década de 1970, se evidencia una reducción de los intervalos que pasaron de un promedio de treinta y ocho años entre el establecimiento de relaciones y la firma de hermanamientos, a un promedio de catorce años, con casos particulares (representados por puntos negros), que serán explicados a continuación por medio de la tabla 1.

\section{Tabla 1. Casos particulares de hermanamientos de ciudades chinas con ciudades de otros países con los que fue posterior el establecimiento de relaciones bilaterales}

\begin{tabular}{|c|c|c|c|c|c|c|}
\hline \multirow{2}{*}{ País } & \multirow{2}{*}{ Continente } & \multirow{2}{*}{ Hermanamiento } & \multirow{2}{*}{$\begin{array}{l}\text { Relaciones } \\
\text { bilaterales }\end{array}$} & \multirow{2}{*}{ Diferencia } & \multicolumn{2}{|c|}{ Ciudades hermanas } \\
\hline & & & & & China & Otro \\
\hline $\begin{array}{l}\text { Bosnia- } \\
\text { Herzegovina }\end{array}$ & Europa & $28 / 05 / 1981$ & 04/03/1995 & $\begin{array}{l}14 \text { años } \\
\text { antes }\end{array}$ & Tianjín & Sarajevo \\
\hline Croacia & Europa & $18 / 06 / 1980$ & $13 / 05 / 1992$ & $\begin{array}{l}12 \text { años } \\
\text { antes }\end{array}$ & Shanghái & Zagreb \\
\hline Eslovenia & Europa & $26 / 10 / 1981$ & $12 / 05 / 1992$ & $\begin{array}{l}11 \text { años } \\
\text { antes }\end{array}$ & Chengdú & Liubliana \\
\hline Macedonia & Europa & $20 / 03 / 1984$ & $12 / 10 / 1993$ & $\begin{array}{l}9 \text { años } \\
\text { antes }\end{array}$ & Nanchang & Skopie \\
\hline Ucrania & Europa & $19 / 10 / 1990$ & 04/01/1992 & $\begin{array}{l}14 \text { meses } \\
\text { antes }\end{array}$ & Wuhan & Kiev \\
\hline Bielorrusia & Europa & $18 / 05 / 1992$ & 20/01/1992 & $\begin{array}{l}4 \text { meses } \\
\text { antes } \\
\end{array}$ & Changchún & Minsk \\
\hline Turkmenistán & Asia & 05/01/1992 & 06/01/1992 & 1 día antes & Lanzhou & Asjabad \\
\hline $\begin{array}{l}\text { Estados } \\
\text { Unidos }\end{array}$ & América & $11 / 02 / 1979$ & 01/01/1979 & $\begin{array}{l}41 \text { días } \\
\text { después }\end{array}$ & Nankín & San Luis \\
\hline Sudáfrica & África & $12 / 06 / 1998$ & 01/01/1998 & $\begin{array}{l}5 \text { meses } \\
\text { después }\end{array}$ & Pekín & Johannesburgo \\
\hline
\end{tabular}

Fuente: Elaboración propia a partir de CIFCA (2015) y Ministerio de Relaciones Exteriores de China (2015b).

Estos casos son relevantes, pues los hermanamientos han sido realizados en $90 \%$ con ciudades capitales. Lamentablemente, no ha sido posible encontrar información sobre los detalles de los hermanamientos o las circunstancias que llevaron a las ciudades chinas a establecer contactos y relaciones con estas ciudades de otros países, con los que la diplomacia estatal no había establecido relaciones oficialmente. Sin embargo, podría existir un indicio de que dichos hermanamientos buscaron sentar las bases de futuras negociaciones diplomáticas.

Por otra parte, otro instrumento importante en el ejercicio de la paradiplomacia son las redes. En cuanto a la participación en redes, vale la pena resaltar que China es uno 
de los países con mayor número de miembros en las redes de ciudades existentes en el mundo. Así, ha participado, entre otras, en la red Ciudades y Gobiernos Locales Unidos, radicada en Barcelona, espacio en el que se ha fortalecido el diálogo con ciudades de otras partes del mundo y se ha puesto en marcha el foro África-China.

Además, los últimos eventos de categoría mundial han puesto a las ciudades chinas en el ojo del mundo, por lo que las autoridades locales "han entendido a la perfección, en los últimos años, el potencial que la organización de grandes eventos tiene para elevar la reputación y la visibilidad internacional de sus ciudades, además de los efectos económicos que los mismos pueden acarrear" (Niño, 2014, p. 18). Estos últimos teniendo mucha mayor relevancia con los lineamientos de política exterior mencionados, toda vez que atraen desarrollo económico e impulsan una imagen de Estado desarrollado y potencia amigable en el escenario internacional.

\section{Conclusiones}

Es evidente la enorme evolución y dinamismo del sistema internacional, principalmente, a partir de la Segunda Guerra Mundial, cuando se evidencia una multiplicación de actores de diferente naturaleza, que interactúan entre ellos y generan una compleja red de relaciones. El surgimiento de los Gobiernos subnacionales como actores en la escena internacional no significa una pérdida de poder y de funciones por parte del Estado central, particularmente con el ejercicio de la paradiplomacia, la cual se presenta como una herramienta de ejercicio del denominado poder blando y que, por tanto, puede traer consigo un fortalecimiento de la presencia internacional de Estado, en nivel donde la alta y tradicional diplomacia no abordaba.

A pesar del "hermetismo" de la República Popular China frente a diversos asuntos en el orden internacional, la actividad internacional de sus ciudades la muestra como un Estado dinámico en el sistema, que no solo atiende asuntos de alta política, sino también asuntos de segundo nivel. Por otra parte, es evidente, como un Estado con aspiraciones de ser un jugador de primer orden en el sistema internacional, que la política exterior china es manejada mediante combinaciones de poder duro y poder blando y tiene lineamientos claros y enfocados a su consolidación como potencia mundial en un escenario multipolar.

La paradiplomacia es un fenómeno que se observa en muchos países y ha respondido a diversas situaciones, tanto en el ámbito internacional como local, que han favorecido su surgimiento y desarrollo. Ya sea por iniciativa de los Estados, ya sea por autonomía de las regiones, las ciudades están involucrándose cada vez más en las relaciones internacionales y han desarrollado instrumentos para institucionalizar su relación con el mundo, fenómeno al que no han sido ajenas las ciudades de la República Popular China.

Es posible considerar la paradiplomacia como una herramienta del poder blando de China; sin embargo, cabe resaltar que no es la única ni la más importante, aunque el 
objetivo no era determinar su grado de importancia, sino analizar la presencia de esta estrategia dentro de las actuaciones de la política exterior de la República Popular China.

Puede asumirse que las ciudades chinas son actores de la política exterior del Estado y serán cada vez más representativas, no solo por sus dinámicas internas de consolidación urbana, crecimiento económico y desarrollo social, sino por el papel que se les ha dado desde el Gobierno central en el proceso de reemergencia de China como potencia.

\section{Referencias}

Alden, C. (2007). China en África. Barcelona: Intermón Oxfam.

ATKearney (2015). Global Cities 2015: The race accelerates. Recuperado de https:// www.atkearney.com/documents/10192/5911137/Global+Cities+201++The+Race+Accelerates.pdf/7b239156-86ac-4bc6-8f30-048925997ac4

Azpíroz, M. L. (2012). Diplomacia pública: el caso de la "guerra contra el terror". Barcelona: UOC.

Bogéa Filho, A. A. M. (2002). A diplomacia federativa. Brasília: Ministério das Relações Exteriores.

Cabestan, J. P. (2010). La politique internationale de la Chine: entre intégration et volonté de puissance. París: Presses de Sciences Po.

Cabrera Toledo, L. (2014). Percepción y geopolítica en la relación Chile-Perú: desde el acta de ejecución hasta la demanda en La Haya. Bloomington, IN: Palibrio.

Calduch Cervera, R. (1993). Dinámica de la sociedad internacional. Madrid: Centro de Estudios Ramón Areces.

Calvento, M., Lorenzo, N. y Herrero, M. S. (2014). Análisis teórico-empírico de la gestión internacional de los actores subnacionales: una propuesta de abordaje para las ciudades intermedias. CONfines de relaciones internacionales y ciencia política, $10(20), 35-58$

Cho, Y. N. y Jeong, J. H. (2008). China's soft power: Discussions, resources, and prospects. Asian Survey, 48(3), 453-472.

China International Friendship Cities Association (CIFCA) (2015). Estadísticas ciudades hermanas. Recuperado de http://www.cifca.org.cn/Web/YouChengTongJi.aspx

Confucius Institute. (2016). Institutos Confucio en el mundo. Recuperado de Confucius Institute Online: http://www.chinesecio.com/m/cio_wci/

Conseil des Communes et Régions d'Europe (CCRE) (2008). Los hermanamientos para el mundo del mañana. Bruselas: CCRE. 
Duchacek, I. D. (1986). The territorial dimension of politics: Within, among, and across nations. Boulder: Westview Press.

Glaser, B. S. y Murphy, M. E. (2009). Soft power with Chinese characteristics. En C. McGiffert (ed.), Chinese soft power and its implications for the United States (pp. 10-26). Washigton, D. C.: Center for Strategic and International Studies.

González Parias, C. H. y Mesa Bedoya, J. C. (2016). Multilateralismo chino, ¿̇giro hacia el revisionismo? El caso del BRICS y el G20. En M. F. Staiano, L. Bogado Bordazar y L. Bono (comps.), Estudios sobre la República Popular China: relaciones internacionales y política interna (pp. 17-32). La Plata: Universidad Nacional de La Plata.

Jain, P. (2005). Japan's subnational governments in international affairs. Oxon, OX: Routledge.

Jiru, S. (1999). Can’t neglect strengthening our country's soft power. Outlook Weekly, 41.

Keating, M. (2001). Paradiplomacia y constitución de redes regionales. Revista Valenciana D'Estudis Autonomics, 36, 39-50.

Keohane, R. O. y Nye, J. S. (1988). Poder e interdependencia: la política mundial en transición. Buenos Aires: Grupo Editorial Latinoamericano.

Lasagna, M. (1996). Cambio institucional y política exterior: un modelo explicativo. Cidob d'Afers Internacionals, 45-64, 45-64 .

León de la Rosa, R. I. (2015). Política exterior china: reconceptualizando el sistema tianxia. En R. I. León de la Rosa y J. C. Gachúz Maya (coords.), Política exterior china: relaciones regionales y cooperación (pp. 19-62). Puebla, México: Universidad Autónoma de Puebla.

Llenderrozas, E. y Finkielsztoyn, M. (2013). Estudios de política exterior: teorías, enfoques y debates. En E. Llenderrozas, Relaciones internacionales: teorías y debates (pp. 167-196). Buenos Aires: EUDEBA.

Mesa Bedoya, J. C., Duarte Herrera, L. K. y González Parias, C. H. (2014). Cooperación internacional descentralizada: estudio sobre su institucionalización en Medellín. Escenarios: empresa y territorio, 3(3), 101-119.

Michelmann, H. J. y Soldatos, P. (1990). Federalism and international relations: The role of subnational units. Oxford: Oxford University Press.

Millán Acevedo, N. (2013). Cambios en las estructuras de poder: interdependencias y asimetrías en la era global. Papel Político, 18(2), 677-699. 
Ministerio de Relaciones Exteriores de China (2003). Política exterior de paz bajo el principio de independencia y autodecisión de China. Recuperado de http://www. fmprc.gov.cn/esp/wjdt/wjzc/t25297.shtml

Ministerio de Relaciones Exteriores de China (2015a). Lista de países que establecieron relaciones diplomáticas con China. Recuperado http://www.fmprc.gov.cn/web/ ziliao_674904/2193_674977/

Ministerio de Relaciones Exteriores (2015b). Misiones diplomáticas en el extranjero. Recuperado de http://www.fmprc.gov.cn/esp/wjb/zwjg/

Niño Pérez, I. (2014). China y la diplomacia pública urbana: caracterización a partir del caso de las grandes ciudades. Ponencia presentada en el $4^{\circ}$ Simposio Electrónico Internacional sobre Política China. Recuperado de http://politica-china. org/imxd/noticias/doc/1394784642Ignacio_Nino_Perez.pdf

Nye, J. S. (1997). China's re-emergence and the future of the Asia-Pacific. Survival, 39(4), 65-79.

Nye, J. S. (2003). La paradoja del poder norteamericano. Madrid: Taurus.

Nye, J. S. (2011). The future of power. Nueva York: Public Affairs.

Paquin, S. (2004). Paradiplomatie et relations internationales: théorie des stratégies internationales des régions face à la mondialisation. Quebec: P.I.E.-Peter Lang.

Pereira, J. C. (2008). Diccionario de relaciones internacionales y política exterior. Barcelona: Ariel.

Pérez Gil, L. V. (2012). Elementos para una teoría de la política exterior. Valencia: Tirant Lo Blanch.

Piñol, À. (2015). La ANC llama a 125.00o personas más para llenar la Meridiana en la Diada. En El País. Recuperado de http://ccaa.elpais.com/ccaa/2015/o9/o4/ catalunya/1441369787_685924.html

Rocha Pino, M. de J. (2006). China en transformación: la doctrina del desarrollo pacífico. Foro Internacional, 46(4), 693-719.

Rodrigues, G. M. A. (2004). Política externa federativa: análise de ações internacionais de estados e municípios brasileiros (Tesis de doctorado, Centro Brasileiro de Relações Internacionais, Río de Janeiro, Brasil).

Rodríguez Aranda, I. y Leiva van de Maele, D. (2013). El soft power en la política exterior de China: consecuencias para América Latina. Polis, 12(35), 497-517. 
Rodríguez Cuadros, M. (2009). Diplomacia y cooperación descentralizada: un instrumento para el desarrollo y la gobernabilidad de las regiones. Gobernanza Global, 1(2), 55-85.

Rodríguez García, M. (2013). Acción directa: hermanamientos, proyectos y otros instrumentos. En M. R. Agost Felip, A. M. Fuertes Eugenio, I. Giménez García y G. Soto Personat (eds.), Cooperación descentralizada pública: introducción, enfoques y ámbitos de actuación (pp. 273-320). Valencia: Universitat Jaume I, Servicio de Publicaciones.

Rosenau, J. N. (1997). Demasiadas cosas a la vez: la teoría de la complejidad y los asuntos mundiales. Nueva Sociedad, 148, 70-83.

Rubiolo, M. F. (2010). Política exterior china hacia los procesos de integración regional de Asean. Astrolabio, 5, 160-184.

Russet, B. y Starr, H. (1989). World politics: The menu for choice. Nueva York: Freeman \& Co.

Sanahuja Perales, J. A. (2007). ¿Un mundo unipolar, multipolar o apolar? El poder estructural y las transformaciones de la sociedad internacional contemporánea. En Cursos de derecho internacional y relaciones internacionales de VitoriaGasteiz (pp. 297-383). Universidad del País Vasco. Recuperado de http://www. ehu.eus/cursosderechointernacionalvitoria/ponencias/pdf/2007/2007_10.pdf

Schiavon, J. A. y Velázquez Flores, R. (2008). Las relaciones exteriores de los Gobiernos locales: un acercamiento teórico-conceptual. En C. Dávila, J. A. Schiavon y R. Velázquez (coords.), Diplomacia local, las relaciones internacionales de las entidades federativas mexicanas (pp. 23-38). México: Universidad Nacional Autónoma de México.

Shixue, J. (2008). The chinese foreign policy perspective. En R. Roett y G. Paz (eds.), China's expansion into the western hemisphere: Implications for Latin America and the United States (pp. 27-43). Washington: Brookings Institution Press.

SiterCities International (2006). Our mission: To promote peace through mutual respect, understanding, and cooperation - one individual, one community at a time. Recuperado de http://www.sister-cities.org/mission-and-history

Sodupe, K. (2002). La estructura de poder del sistema internacional: del final de la Segunda Guerra Mundial a la posguerra fría. Madrid: Fundamentos.

Strange, S. (1998). States and markets (2. ${ }^{\mathrm{a}}$ ed.). Londres: Pinter Publishers. 
Vigevani, T. (2004). El marco jurídico e institucional para la gestión internacional de los actores subnacionales gubernamentales de Brasil. Integración y Comercio, $8(21), 27-46$.

Vigevani, T. y Figueiredo B. Prado, D. (2012). Acciones y problemas de la paradiplomacia en Brasil. En L. Maira (comp.), La política internacional subnacional en América Latina (pp. 177-208). Buenos Aires: Zorzal.

Wang, H. (2000). Multilateralism in Chinese foreign policy: The limits of socialization. Asian Survey, 40(3), 475-491.

Zaharna, R. S. (2009). Mapping out a spectrum of public diplomacy initiatives. information and relational communication frameworks. En N. Snow y P. M. Taylor (eds.), Routledge handbook of public diplomacy (pp. 86-100). Nueva York: Routledge.

Zeraoui, Z. (2011). Diplomacia paralela y las relaciones internacionales de las regiones. Desafíos, 23(1), 59-96.

Zhongying, P. (1997). Soft power in international relations and others. Strategy and Management, 2, 49-51. 